\title{
Sociodemographic Characteristics and Aetiological Factors of Vaginal Discharge in Pregnancy
}

\author{
Omole-Ohonsi A* FWACS \\ Nwokedi E.E.** FMCPath \\ *Department of Obstetrics and Gynaecology, **Department of Microbiology, \\ Bayero University/Aminu Kano Teaching Hospital, Kano, Nigeria.
}

Correspondence to: Dr. Omole-Ohonsi A, P. O. Box 14578 ,

General Post Office, Kano, Nigeria. Tel No. +234803 7870540. Email: aomohonsi@yahoo.com

Synopsis: Determining the aetiological factors of vaginal discharge will prevent erroneous empirical treatment Word count:2724

Key words: Vaginal discharge, aetiological factors, empirical treatment, pregnancy, Kano-Nigeria.

\begin{abstract}
Objective: This is a study on the aetiology of vaginal discharge among pregnant women in Kano, Northern Nigeria.

Method: This longitudinal descriptive study was conducted at Aminu Kano Teaching Hospital, Kano, Nigeria, between $1^{\text {st }}$ January 2004 and $31^{\text {st }}$ December 2004. The pregnant women who had vaginal discharge among those who attended the antenatal clinic during the period of study were recruited.

Results: The prevalence of vaginal discharge in pregnancy in Kano, Northern Nigeria, was 1 in 20 antenatal patients. Infective vaginal discharge occurs with similar frequency to that of non-infective origin. The prevalence of infective vaginal discharge was found to decrease with increasing age, parity and Western education, and increased among patients who had Human immunodeficiency virus (HIV) infection and diabetes mellitus in pregnancy.
\end{abstract}

Conclusion: We recommend close surveillance among high risk patients, and also the aetiology of vaginal discharge should be determined, so that appropriate treatment can be offered in order to prevent avoidable feto-maternal morbidity and mortality from erroneous empirical treatment.

Key words: Vaginal discharge, aetiological factors, empirical treatment, pregnancy.

\section{Introduction}

Any secretion which comes out from the vagina apart from blood is called vaginal discharge $[1,2]$. It is defined as any amount of secretion that the patient is worried about [3]. The discharge may be normal (physiological) or abnormal (pathological) [1-4]

Vaginal discharge increases normally in pregnancy due to increasing levels of serum estrogen influence and blood flow to the reproductive organs, with rapid turnover of cells [1-3]. Vaginal discharge is heavier and will have more cervical mucus in it as the gestational age increases and as labour approaches [1, 2]. This normal (physiological) vaginal discharge is colourless or white, non-irritating and odourless, or has mild odour [1-3]. It is non-infective in nature with no sequel [1-3]. Self-medication and the use of tampons should be avoided as they could alter the normal floral balance or introduce infection into the vaginal canal, with resultant vaginal infection (vaginitis)[ 1,2$]$. This is why when patients notice vaginal discharge in pregnancy, they should be told to use cotton panty liners if they are uncomfortable, and to inform their Doctors[1-3], so that the aetiology could be determined, rather than embarking on self-medication, which could introduce new infections into the vagina when leucorrhoea is erroneously treated $[1,2]$.

Abnormal (Pathological) vaginal discharge on the other hand may be green, yellow, brown or red in colour, and may be foul smelling, with accompanied itching or soreness in the genital area[1,3-5]. These pregnant women will also need to contract their Doctors, because these may be signs of vaginitis or sexually transmissible infections [1-7]. Other entities that may present with vaginal discharge include retrograde follow of 
semen, stress incontinence, drainage of liquor in preterm rupture of fetal membranes, preterm labour with passage of cervical mucus plug, or malignancy $[1,2]$. Delay or non-treatment of these patients may lead to serious complications, which may be associated with poor fetomaternal outcome $[1,2]$

No study has been done in our centre on this subject, and it is against this background, that this study on the aetiology of vaginal discharge among pregnant women in Kano, Northern Nigeria, was conceived in order to determine the causes, and to make recommendations on how to manage them.

\section{Patients and Methods}

This descriptive study was conducted at Aminu Kano Teaching Hospital, Kano, Nigeria, between $1^{\text {st }}$ January 2004 and $31^{\text {st }}$ December 2004. The pregnant women who had vaginal discharge among those who attended the antenatal clinic during the period of study were recruited. This study was exempted from our Institutional Review Board approval because there is no intervention, and it is an observational study.

A high vaginal swab was collected with a sterile swab stick, from each of the pregnant women with vaginal discharge by a Consultant Obstetrician using the appropriate techniques. The swab sticks were labelled and sent immediately to the laboratory for immediate plating on MacConkey, chocolate and saboraud dextrose agar, and incubated aerobically overnight at $37^{\circ} \mathrm{C}$. The chocolate agar was incubated in 5\% carbondioxide atmosphere (candle jar). Stroke disc diffusion technique was used for antimicrobial sensitivity test. Examination of the samples was done to identify the microbial organisms and their antibiotic sensitivities.

All our antenatal women had pre-test counseling for human immunodeficiency virus (HIV) infection at booking, and almost all of them agreed to testing. Screening was done using HIV 1 and 2 stat pak assay and Rapid enzyme immunoassay Genie II for HIV 1 and 2 antibody detection. Where further confirmation was required, immunoconfirm for HIV 1 and 2 antigen detection was used, which test for $\mathrm{p}^{24}$ (core antigen), $\mathrm{p}^{31}$ (endonucleus polymerase antigen), $\mathrm{gp}^{41}$ (envelope antigen). This enabled us to determine the HIV status of virtually all our antenatal women.

All our antenatal women had urinalysis done for glucose and protein at booking and at each follow up visit. Those with glycosuria on one occasion before and two occasions after 16 weeks of gestation together with the potential diabetics, had oral glucose tolerance test (OGTT) done, which enabled us to determine all the antenatal women with diabetes mellitus in pregnancy.

The results that were obtained were presented as mean and standard deviation. Quantitative data were expressed as percentages, and recorded using tables. Statistical analyses of the data were performed using a chi-square test with or without linear trend (Mantel extension), and a P-value of $<0.05$ was regarded as significant.

\section{Results}

During the period of study, 3143 pregnant women attended the antenatal clinic. Among them 160 women were confirmed to have vaginal discharge, giving a prevalence of 1 in 20 antenatal patients for vaginal discharge in Kano.

$54 \%$ of the patients had vaginal discharge of infective origin, while $46 \%$ had that of noninfective origin. There was no statistically significant difference in the prevalence of infective and non-infective vaginal discharge among the pregnant women $\left(\mathrm{X}^{2}=0.78\right.$, df $\left.=1, \mathrm{P}>0.05\right)$. Leucorrhoea accounted for $85 \%$ of the cases of vaginal discharge of non-infective origin, while retrograde flow of spermatozoa was found in $8 \%$ of the cases, prelabour rupture of the fetal membranes $(4 \%)$, premature labour $(3 \%)$. There was no case of associated genital tract malignancy or stress incontinence.

Among those with vaginal discharge of infective origin, Candida albicans were isolated in $94 \%$ of the cases, while staphylococcus aureus was isolated in 6\% (Table I).

The age range of the patients was between 15 and 42 years, with a mean age of $27.1 \pm 7.7$. Age was found to show statistically significant difference on the risk of exposure $\mathrm{X}_{\text {trend }}^{2}=30.427, \mathrm{P}=0.000$. The exposure to vaginal infection showed a bimodal trend. It decreased with increasing age up to 40 years, and thereafter started increasing among the 40-44 years age group, while the reverse was the case for non-infective vaginal discharge (Table II A).

The parity range was from 0 to 12 , with a mean parity of $3.5 \pm 3.5$. Parity showed statistically significant difference on the risk of exposure $\mathrm{X}_{\text {trend }}^{2}$ $=19.351, \mathrm{P}=0.00001$. The exposure to vaginal infection showed a linear trend. It decreased with increasing parity, while the reverse was the case with non-infective vaginal discharge (Table II B). 
Educational status showed statistically significant difference on the risk of exposure $\mathrm{X}_{\text {trend }}^{2}=4.105, \mathrm{P}=$ 0.04275 . The exposure to vaginal infection was higher among those with no form of education, followed by those with Qur'anic education only, and the least was among those with Western education. The reverse was the case among those with non-infective vaginal discharge (Table II C).

$12 \%$ of the patients with vaginal discharge were HIV-positive. Among them, 83\% had vaginal discharge of infective origin (positive microbial isolates), while $17 \%$ had that of non-infective origin. HIV-status showed statistically significant difference on the risk of exposure. $X^{2}=5.86, \mathrm{df}=1$, $\mathrm{P}<0.05$ (Table III).

$6 \%$ of the pregnant women who had vaginal discharge had diabetes mellitus. Among them, 89\% had vaginal discharge of infective origin, while $11 \%$ had that of non-infective origin. Diabetes mellitus showed statistically significant difference on the risk of exposure. Fisher exact test $=0.03, \mathrm{P}<$ 0.05 (Table IV).

Table I: Causes of Vaginal Discharge



Table II: Socio-demographic Characteristics of the Pregnant Women.

\begin{tabular}{l|l|l|l}
\multicolumn{4}{c}{ A: Age } \\
\hline $\begin{array}{l}\text { Age in } \\
\text { Years }\end{array}$ & $\begin{array}{l}\text { Positive } \\
\text { cultures }\end{array}$ & $\begin{array}{l}\text { Negative } \\
\text { Cultures }\end{array}$ & Total \\
\hline 1519 & $28(78 \%)$ & $8(22 \%)$ & $36(100 \%)$ \\
$20-24$ & $26(68 \%)$ & $12(32 \%)$ & $38(100 \%)$ \\
$25-29$ & $16(62 \%)$ & $10(38 \%)$ & $26(100 \%)$ \\
$30-34$ & $9(39 \%)$ & $14(61 \%)$ & $23(100 \%)$ \\
$35-39$ & $4(18 \%)$ & $18(82 \%)$ & $22(100 \%)$ \\
$40-44$ & $3(20 \%)$ & $12(80 \%)$ & $15(100 \%)$ \\
\hline \multicolumn{4}{c}{ Mean age $=27.1 \pm 7.7$} \\
$\mathrm{X}_{\text {trend }}^{2}=30.427, \mathrm{P}=0.00000$ (statistically significant)
\end{tabular}

\begin{tabular}{l|l|l|l}
\multicolumn{1}{c}{ B: Parity } & $\begin{array}{l}\text { Positive } \\
\text { Cultures }\end{array}$ & $\begin{array}{l}\text { Negative } \\
\text { Cultures }\end{array}$ & Total \\
\hline Parity & $43(80 \%)$ & $11(20 \%)$ & $54(100 \%)$ \\
& Primigravidae (0) & $32(56 \%)$ & $57(100 \%)$ \\
Multigravidae (1-4) & $25(44 \%)$ & $31(63 \%)$ & $49(100 \%)$ \\
Grand multiparae( $\geq 5)$ & $18(37 \%)$ & &
\end{tabular}
Diabetes Mellitus

\begin{tabular}{l|l|l|l}
\hline $\begin{array}{l}\text { Diabetes } \\
\text { Mellitus }\end{array}$ & $\begin{array}{l}\text { Positive } \\
\text { Cultures }\end{array}$ & $\begin{array}{l}\text { Negative } \\
\text { Cultures }\end{array}$ & Total \\
\hline Positive & $8(89 \%)$ & $1(11 \%)$ & $9(100 \%)$ \\
Negative & $78(52 \%)$ & $73(48 \%)$ & $151(100 \%)$ \\
Total & 86 & 74 & 160 \\
\hline \multicolumn{2}{l}{ Fisher exact test $=0.03(\mathrm{P}<0.05)$ statistically significant }
\end{tabular}

\section{Discussion}

The incidence of vaginal discharge in pregnancy in this study was 1 in 20 antenatal patients, which is similar to the findings in other studies from developing nations [1-3].

The reason for the similar finding in these studies is because, majority of vaginal discharge in pregnancy has similar aetiology, which are the increasing hormonal influence and blood supply to the genital organs as the gestational age increases until delivery 1-3]. They result in leucorrhoea, or predispose them to vaginitis with infective vaginal discharge due to alteration in vaginal $\mathrm{pH}$ [1-3]. This common pathophysiology, accounted for the reason why there was no statistical difference in the prevalence of vaginal discharge of infective and non-infective origin among the pregnant women.

The commonest cause of vaginal discharge of noninfective origin was leucorrhoea $(85 \%)$, while for infective vaginal discharge it was Candida albicans (94\%). This is the same findings in other reviews. ${ }^{1-}$ ${ }^{3,8}$ Leucorrhoea is a normal effect of pregnancy [13 ], and the cervix produces increased fluid during pregnancy that helps to keep the vaginal canal clean and infection free [1-3]. The increased prevalence of Candida albicans, a vaginal commensal, is because of the increased acidity in the vaginal canal in pregnancy, which acts as a physiological barrier 
to protect the fetus in utero [2, 8-10]. Candida albicans is one of the very few organisms that can survive in this hostile environment, and it proliferates to become pathogenic $[9,10]$.

Other causes of non-infective vaginal discharge that were found in this study were retrograde flow of semen ( $8 \%$ ), slowly leaking preterm rupture of the fetal membranes (4\%), and premature labour $(3 \%)$, which has also been reported by several authors[1-3], and it informs the need for proper search for the cause of the vaginal discharge, because failure to make the diagnosis of preterm labour/rupture of fetal membranes could result in avoidable feto-maternal morbidity and mortality[1-3]. With retrograde flow of semen, treatment could lead to introduction of infection [13].

Maternal age was found to influence the type of vaginal discharge. Teenagers were found to have more cases of vaginal discharge of infective origin, despite the sedative effect of progesterone on the libido in these young mothers in pregnancy, which reduce or even abolish sexual urge and coital frequency, probably because of inexperience about personal and environmental hygiene [1-3]. The risk of exposure to vaginal infection in this study decreased with increasing age up to 40 years, because of maturity and improved personal and environmental hygiene[1-3]. The increase in the risk of exposure to infective vaginal discharge among the 40-44 years age group, was probably because of increased coital frequency resulting from relative increase in serum androgen levels, and reduction in the physiological barrier in the vaginal canal due to relatively low estrogen levels as these women enter into the climacteric period[13]. The reverse was the case with non-infective vaginal discharge for the same reasons. These same reasons accounted for why the exposure to vaginal infection decreased with increasing parity, and that of non-infective vaginal discharge increased with increasing parity, which is compatible with the findings of other authors [1-9].

The risk of exposure to vaginal infection was highest among those who had no form of education followed by those with only Qur'anic education, and least among those with Western education. This could be due to higher level of sophistication, enlightenment and utilization of orthodox medicine among those with Western education. This concurs with the findings of other authors [2, $3,9]$. Those who lack Western education patronize traditional medicine more [2]. This high patronage of traditional medicine which involve insertions into the vagina under aseptic conditions predispose them to vaginal discharge of infective origin[3,9].

HIV positive and diabetic pregnant women had more cases of vaginal discharge of infective origin, and Candida albicans was isolated in all the cases, which is also the findings in others studies $[1,11]$. The high prevalence of infective vaginal discharge among the HIV positive and diabetic women, was because Candida albicans is an opportunistic commensal in the vagina, which proliferate to become pathogenic in immuno-suppressed individuals like HIV positive women [1, 11], or when other commensals are eliminated by the higher acidity in the vaginal canal, due to the higher vaginal epithelial glycogen content in patients with diabetes mellitus $[2,3,9,10]$. This is similar to the findings in other studies [8-11].

\section{Conclusion and Recommendations:}

The prevalence of infective and non-infective vaginal discharge among the pregnant women was similar in this study.

Young maternal age, low parity, lack of Western education, HIV infection and diabetes mellitus were found to predispose the pregnant women to vaginal discharge of infective origin, and should have closer surveillance.

All cases of vaginal discharge in pregnancy should be investigated properly in order to determine the aetiology, so as to avoid complications from erroneous empirical treatments.

\section{References}

1. Paranipe Aisha: Vaginal discharge during pregnancy. India Parenting. 2004:35-39

2. Hunter $\mathrm{H} \mathrm{H}$ : Vaginal discharge and bleeding in pregnancy. Baby World. 2005:5-10

3. Asem A M: Vaginal discharge in pregnancy- what is normal? Pregnancy birth. 2002:21-27

Otubu J A M, Sagay A S, Imade G, Towobola O A, Uguru V E: Sexually transmitted disease amongst consecutive attendance at antenatal 